\title{
The Emergence of Invasive
} Streptococcus pneumoniae Serotype 24F in Lebanon: Complete Genome Sequencing Reveals High Virulence and Antimicrobial Resistance Characteristics

\section{OPEN ACCESS}

Edited by:

Axel Cloeckaert

l'Alimentation et l'Environnement (INRAE), France

Reviewed by:

Stephanie Lo,

Wellcome Sanger Institute (WT), United Kingdom

Annalisa Pantosti,

National Institute of Health (ISS), Italy

Kwan Soo Ko,

Sungkyunkwan University School of

Medicine, South Korea

${ }^{*}$ Correspondence: Ibrahim Bitar

ibrahimbitar5@gmail.com

Ghassan Dbaibo

gdbaibo@aub.edu.lb

Specialty section

This article was submitted to Antimicrobials, Resistance

and Chemotherapy,

a section of the journal

Frontiers in Microbiology

Received: 04 December 2020 Accepted: 29 January 2021

Published: 19 February 2021

Citation:

Reslan L, Finianos M, Bitar I, Moumneh MB, Araj GF, Zaghlout A,

Boutros $C$, Jisr T, Nabulsi M,

Kara yaccoub G, Hamze M,

Osman M, Bou Raad E, Hrabak J,

Matar GM and Dbaibo G (2021) The

Emergence of Invasive Streptococcus

pneumoniae Serotype 24F

in Lebanon: Complete Genome

Sequencing Reveals High Virulence

and Antimicrobial Resistance

Characteristics.

Front. Microbiol. 12:637813.

do: 10.3389/fmicb.2021.637813
Lina Reslan', Marc Finianos², Ibrahim Bitar2*, Mohamad Bahij Moumneh', George F. Araj ${ }^{1,3}$, Alissar Zaghlout', Celina Boutros' ${ }^{1}$, Tamima Jisr ${ }^{4}$, Malak Nabulsi ${ }^{5}$, Gilbert Kara yaccoub ${ }^{5}$, Monzer Hamze ${ }^{6}$, Marwan Osman 7 , Elie Bou Raad ${ }^{7}$, Jaroslav Hrabak', Ghassan M. Matar ${ }^{1,8}$ and Ghassan Dbaibo ${ }^{1,9 *}$

\begin{abstract}
${ }^{1}$ Center for Infectious Diseases Research (CIDR) and WHO Collaborating Center for Reference and Research on Bacterial Pathogens, American University of Beirut, Beirut, Lebanon, ${ }^{2}$ Department of Microbiology, Faculty of Medicine and University Hospital in Plzen, Charles University, Plzen, Czechia, ${ }^{3}$ Department of Pathology and Laboratory Medicine, American University of Beirut Medical Center, Beirut, Lebanon, ${ }^{4}$ Department of Laboratory and Blood, Makassed General Hospital, Beirut, Lebanon, ${ }^{5}$ Laboratory, Haykal Hospital, Tripoli, Lebanon, ${ }^{6}$ Department of Microbiology, Nini Hospital, Tripoli, Lebanon, ${ }^{7}$ El-Youssef Hospital Center, Department of Clinical Laboratory, Halba, Lebanon, ${ }^{8}$ Department of Experimental Pathology, Immunology, and Microbiology, Faculty of Medicine, American University of Beirut, Beirut, Lebanon, ${ }^{9}$ Department of Pediatrics and Adolescent Medicine, Faculty of Medicine, American University of Beirut, Beirut, Lebanon
\end{abstract}

Background: Invasive pneumococcal disease (IPD) remains a global health problem. IPD incidence has significantly decreased by the use of pneumococcal conjugate vaccines (PCV). Nevertheless, non-PCV serotypes remain a matter of concern. Eight Streptococcus pneumoniae serotype 24F isolates, belonging to a non-PCV serotype, were detected through the Lebanese Inter-Hospital Pneumococcal Surveillance Program. The aim of the study is to characterize phenotypic and genomic features of the $24 \mathrm{~F}$ isolates in Lebanon.

Methods: WGS using long reads sequencing (PacBio) was performed to produce complete circular genomes and to determine clonality, antimicrobial resistance and virulence determinants.

Results: The sequencing results yielded eight closed circular genomes. Three multilocus sequence typing (MLST) types were identified (ST11618, ST14184, ST15253). Both MLST and WGS analyses revealed that these isolates from Lebanon were genetically homogenous belonging to clonal complex CC230 and clustered closely with isolates originating from Canada, United States of America, United Kingdom and Iceland. Their penicillin binding protein profiles correlated with both $\beta$-lactam susceptibility patterns and MLST types. Moreover, the isolates harbored the macrolide and tetracycline resistance genes and showed a similar virulence gene profile. To our knowledge, this study represents the first report of complete phenotypic and genomic characterization of the emerging Streptococcus pneumoniae, serotype 24F, in the Middle East and North Africa region.

Keywords: Streptococcus pneumoniae, serotype $24 \mathrm{~F}$, whole-genome sequencing, Lebanon, antibiotic resistance 


\section{INTRODUCTION}

Streptococcus pneumoniae is a major causative agent responsible for severe infections mainly among young children, elderly individuals and those with chronic illnesses and immunodeficiency disorders (Global Burden of Disease Study, 2018; Principi et al., 2018). The World Health Organization (WHO) estimated that pneumococcal disease is responsible for 1.6 million deaths every year, with 0.7 million to 1 million being children younger than 5 years, mostly in developing countries (World Health Organization, 2007).

Based on the capsular polysaccharide structure, almost 100 pneumococcal serotypes have been identified so far (Ganaie et al., 2020). These serotypes vary in terms of incidence, antibiotic resistance, and disease manifestation (Hausdorff et al., 2005) and only a limited number of them cause the majority of invasive pneumococcal disease (IPD) (Namkoong et al., 2016). Introduced for adults in 1983, the 23-valent pneumococcal polysaccharide vaccine (PPV23), targeting 23 serotypes represents 85-90\% of all serotypes causing IPD (Pitsiou and Kioumis, 2011). The pneumococcal conjugate vaccine (PCV) that covers seven serotypes also known as PCV7 (4, 6B, 9 V, 14, 18C, 19F, and $23 \mathrm{~F})$ was implemented as a routine vaccination in 2000 and was replaced by PCV10 with three additional serotypes $(1,5$, and $7 F)$ followed by PCV13 covering six additional serotypes from PCV7 $(1,5,7 \mathrm{~F}, 3,6 \mathrm{~A}$, and 19A). Those vaccines are recommended for children under the age of 2 (Daniels et al., 2016; Isturiz et al., 2018). The pediatric use of PCV13 has led to sharp declines in pneumococcal disease among unvaccinated adults and children, which led the Advisory Committee on Immunization Practices (ACIP) to reevaluate its use in the United States for adults and recommend its administration for adults aged $\geq 65$ years with only underlying medical conditions (Matanock et al., 2019). In Lebanon, PCV7 was introduced by the private sector, in 2006, followed by PCV10 and PCV13 in 2010. As of January 2016, PCV13 has been added to the expanded program of Immunization using the $2++1$ schedule. However, there are no official national recommendations for adult immunization, and limited only to some practitioners following the international guidelines in their private practice.

The PCVs introduced has proven to be very effective against vaccine serotypes causing IPD. Yet, this reduction is partly offset by an increase in non-vaccine serotypes (NVT), known as serotype replacement that might be occurring through mainly two mechanisms (Weinberger et al., 2011). The first is expansion of non-vaccine-type lineages to partly occupy the niche vacated by vaccine-type lineages and the second is the expansion of preexisting clones of non-vaccine serotypes within the same lineage to replace vaccine-type serotypes after PCV13 introduction (Croucher et al., 2013). The level of antibiotic consumption undoubtedly plays a role in inducing a selective pressure on pneumococcal strains from nasopharyngeal microbiota and certainly influences serotype distribution in different countries (Lo et al., 2019)Additionally, elevated recombination rates in this species within its locus may drive the change of serotype through a process known as "serotype switching"(Ansaldi et al., 2011) for instance serotype
19A donors and recipients belonging to serotype 4 (Hicks et al., 2007) or serotype 9V and 6A (Vestrheim et al., 2012). Furthermore, vaccine schedules and vaccine coverage can also affect the evolution of pneumococcal epidemiology.

Most of the published data about pneumococcal disease from the Middle East and North Africa (MENA), including Lebanon, focused on the incidence of IPD, the serotypes distribution, and the vaccine coverage (Hanna-Wakim et al., 2012; Mokaddas and Albert, 2012; Taha et al., 2012; Al-Sheikh et al., 2014; Bahy et al., 2016; Moghnieh et al., 2018, 2020). In Lebanon, the Lebanese Inter-hospital Pneumococcal Surveillance program (LIPSP), serves as a national prospective surveillance program, established in 2005 in collaboration with the Lebanese Ministry of Public Health. Previously, we reported data from LIPSP, collected from October 2005 till December 2011, and identified a total of 257 isolates of invasive S. pneumoniae. The vaccine coverage was 41.4, 53.9, and 67.2\% for PCV7, PCV10, and PCV13 serotypes, respectively, among all age groups; for patients $<2,2-$ 5 , and $>60$ years of age, PCV7 coverage was 50, 51, and 35\%, respectively, PCV10 coverage was 53, 74, and 45\%, respectively, and PCV13 coverage was 63, 80, and 68\%, respectively (HannaWakim et al., 2012). In countries that introduced PCV-10 or PCV-13 on their immunization schedules, an increase related to non-vaccine serotypes (NVTs) has been observed (Balsells et al., 2017). To date, reported increases in NVTs in the MENA are largely lacking. In Europe and the Western Pacific regions, but not in North America, serotype $24 \mathrm{~F}$ has been reported as one of the emerging NVTs (Balsells et al., 2017). In France, a rebound in pneumococcal meningitis due to the strong emergence of serotype $24 \mathrm{~F}$, frequently penicillin-resistant, was observed in a 16 year French nationwide population-based study, especially during 2015 and 2016 (Ouldali et al., 2018). The 24F group serotype was found to be responsible for several instances of penicillin non-susceptible related IPD in Catalonia, Spain (Munoz-Almagro et al., 2011). In addition, it has been associated with macrolide-lincosamide and tetracycline resistance (Janoir et al., 2016). In Lebanon, LIPSP ongoing surveillance has recently revealed an increase in the number of $24 \mathrm{~F}$ isolates, with 4 isolates in 2019.

Neither the available vaccines PPV or PCV nor the ones being developed (PCV15 and PCV20) covers the 24F serotype, therefore, it is crucial to monitor the susceptibility and epidemiology of this serotype. Thus, the current study aims to characterize S. pneumoniae serotype $24 \mathrm{~F}$ isolates among IPD cases in Lebanon.

\section{MATERIALS AND METHODS}

\section{Isolate Collection}

Eight S. pneumoniae isolates, belonging to serogroup 24, were collected among a total of 587 isolates through the Lebanese Inter-hospital Pneumococcal Surveillance program (LIPSIP), a prospective surveillance program, established in 2005 and still ongoing. The study included samples collected from 78 hospitals across Lebanon (Beirut, North and South Lebanon, Mount Lebanon and Bekaa) including patients of all age groups, 
diagnosed with IPD. When identifying pneumococcus from blood, CSF, or other sterile sites, hospitals alert the surveillance coordinator. Within $24 \mathrm{~h}$, samples are collected by the courier, subcultured, and frozen after proper labeling and identification. Demographic data and clinical information were collected for all isolates. All the isolates were stored at $-80^{\circ} \mathrm{C}$ till tested.

\section{Identification and Antibiotic Susceptibility Testing (Phenotypic Tests)}

Bacterial isolates were cultured on Mueller-Hinton blood sheep agar (MHSB) plates and incubated at $37^{\circ} \mathrm{C}$ with $5 \%$ $\mathrm{CO}_{2}$ for $24 \mathrm{~h}$. Identification of each isolate was done using colonies morphology, optochin susceptibility test and confirmed using matrix-assisted laser desorption ionization-time of flight mass spectrometry (MALDI-TOF MS) using MALDI Biotyper software (Brucker Daltonics, Bremen, Germany). Antibiotic susceptibility testing was processed according to $\mathrm{VITEK}^{\circledR}$ 2 platform manufacturer's instructions. Briefly, a bacterial suspension using $0.45 \%$ sodium chloride solution was adjusted to an optical density of $0.5-0.63$ McFarland units. The VITEK2 AST-ST03 test card (bioMérieux, France) was inoculated with the bacterial suspension. The cards were loaded into the VITEK $^{\circledR}-2$ system and results were automatically reported by VITEK $^{\circledR}-2$ Software release 8.01. Antibiotic agents tested include: chloramphenicol (C), tetracycline (T), sulfamethoxazoletrimethoprim (SXT), vancomycin (Va), erythromycin $(\mathrm{E})$, clindamycin (DA), levofloxacin (LVX), and oxacillin (Ox), as recommended by the Clinical and Laboratory Standards Institute (Clinical and Laboratory Standards Institute [CLSI], 2019). For oxacillin resistant isolates, E- tests were additionally performed for both penicillin (PEN) and ceftriaxone (CRO). For all isolates other than those from CSF, PEN susceptibility and resistance refer to PEN MIC of $\leq 2$ and $\geq 8 \mu \mathrm{g} / \mathrm{ml}$, respectively. Moreover, isolates with ceftriaxone (CRO) $\mathrm{MIC} \leq 1 \mu \mathrm{g} / \mathrm{mL}$ were considered susceptible while those with $\mathrm{MIC} \geq 4$ were resistant. For CSF isolates, PEN susceptibility and resistance refer to PEN MIC of $\leq 0.06$ and $\geq 0.12 \mu \mathrm{g} / \mathrm{ml}$, respectively, while for $\mathrm{CRO}, \mathrm{MIC} \leq 0.5 \mu \mathrm{g} / \mathrm{mL}$ were considered susceptible while those with $\mathrm{MIC} \geq 2$ were resistant. In isolates that showed intermediate and/or resistant phenotype were defined as non-susceptible. At the molecular level, all isolates were species identified using the $16 \mathrm{~S}$ rRNA gene sequencing useful to identify the S. pneumoniae based on the location of cytosine at the 203 position (Arbique et al., 2004).

\section{Whole-Genome Sequencing (WGS) and Annotation}

NucleoSpin Microbial DNA kit (Macherey-Nagel, Germany) was used to extract the genomic DNA of the eight strains. The extracted DNA was then subjected to shearing using g-tubes (Covaris, United States). Genomic libraries were prepared according to the microbial multiplexing protocol according to the manufacture instructions without size selection. Sequel I platform (Pacific Biosciences, California, United States) was used for long reads sequencing. The Microbial Assembly pipeline featured in SMRT Link v8.0 was used to perform the assemblies of the genomes with the default minimum seed coverage (30X). ResFinder 3.2 (Zankari et al., 2012), CARD (Alcock et al., 2020), PlasmidFinder (Carattoli et al., 2014), VirulenceFinder 2.0 (Kleinheinz et al., 2014), and VFDB (Liu et al., 2019), ISfinder database, and MLST 2.0 (Larsen et al., 2012) were used to detect antibiotic resistance genes, plasmid replicon type, virulence genes, mobile elements and multilocus sequence types (STs), respectively. RAST 2.0 combined with BLASTP/BLASTN were used to predict open reading frame (ORF) (Brettin et al., 2015). Mauve v.2.3.1 was used to perform comparative genome alignments while diagrams and gene organization were sketched using Inkscape 0.92.4.

\section{Molecular Characterization of Their Capsular Genes}

A multiplex-PCR assay was performed to detect isolates' serotypes according to the Center for Disease Control's protocol. Briefly, seven multiplex PCR reactions were run including 38 primer pairs, each pair corresponding to a specific serotype ${ }^{1}$. DNA of CDC S. pneumoniae isolates of known serotypes were used as positive controls. PCR reactions were run in Bio-Rad $\mathrm{C} 1000^{\mathrm{TM}}$ Thermal Cycler C1000, then the products were run on a $1 \%$ agarose gel. Finally, the obtained bands were compared to their corresponding positive controls.

Additionally, all the isolates were checked for 92 capsular genes (CR931632-CR931722, JF911515.1 and HV580364.1) (Bentley et al., 2006; Kapatai et al., 2016). FASTA files were downloaded from NCBI nucleotide database using BLAST $^{\circledR}+$ v.2.10.1 (Camacho et al., 2009). Serogroup/type 24F identification was performed through detection of associated genes with a cut-off of $80 \%$ coverage query and a $95 \%$ sequence identity (Kapatai et al., 2016).

\section{Multilocus Sequence Typing (MLST)}

MLST was performed to determine the sequence type (ST) for all the $S$. pneumoniae strains by uploading the sequences to the PubMLST Database. Sequence types (STs) and assignment to clonal complex (CC) was performed using PHYLOViZ 2.0 program. STs sharing at least five allelic variants composed a CC (Mayanskiy et al., 2017).

\section{Phylogenetic Analysis}

Forty-six S. pneumoniae genomes of serogroup 24 were downloaded from PubMLST as references representing 86 downloaded complete and draft genomes along with our eight complete genomes. These strains were phylogenetically clustered using core genome single-nucleotide polymorphisms (SNPs) by parsnp v1.2, available in the Harvest suite (Treangen et al., 2014) using the S. pneumoniae R6 (AE007317.1) as reference. SNPs identified in local collinear blocks were subsequently used for reconstructing an approximate maximum-likelihood tree using FastTree 2 (Price et al., 2010) while including the general time reversible (GTR) model of nucleotide substitution. The

\footnotetext{
${ }^{1}$ https://www.cdc.gov/streplab/downloads/pcr-us-clinical-specimens.pdf
} 
TABLE 1 | Clinical and demographic characteristics of the invasive pneumococcal isolates of serotype 24F in Lebanon.

\begin{tabular}{|c|c|c|c|c|c|c|c|c|}
\hline \multirow[t]{2}{*}{ Isolate } & \multicolumn{7}{|c|}{ Clinical data } & \multirow[t]{2}{*}{ Serotype } \\
\hline & Year & Origin & Sex & Age $(\mathrm{m} / \mathrm{y})$ & Sample source & Diagnosis & Outcome & \\
\hline 310 & 2013 & MGH-Beirut & Female & $3 \mathrm{~m}$ & blood & Bacteremia & N/A & $24 \mathrm{~F}$ \\
\hline 475 & 2017 & Haykel hospital- North LBN & Female & $8 \mathrm{~m}$ & CSF & Meningitis & Death & $24 \mathrm{~F}$ \\
\hline 521 & 2017 & Nini hospital- North LBN & Female & $5 y$ & blood & Bacteremia & $\mathrm{N} / \mathrm{A}$ & $24 \mathrm{~F}$ \\
\hline 525 & 2018 & El Youssef hospital-North LBN & Male & 44 y & pleural fluid & Pneumonia & $\mathrm{N} / \mathrm{A}$ & $24 \mathrm{~F}$ \\
\hline 563 & 2019 & Haykel hospital- North LBN & Male & $6 y$ & blood & Pneumonia & Recovered & $24 \mathrm{~F}$ \\
\hline 566 & 2019 & AUBMC-Beirut & Male & $3 y$ & blood & Bacteremia & Recovered & $24 \mathrm{~F}$ \\
\hline 573 & 2019 & MGH- Beirut & Male & $2 y$ & blood & Pneumonia & $\mathrm{N} / \mathrm{A}$ & $24 \mathrm{~F}$ \\
\hline 574 & 2019 & MGH-Beirut & Male & $5.5 \mathrm{y}$ & blood & Meningitis & $\mathrm{N} / \mathrm{A}$ & $24 \mathrm{~F}$ \\
\hline
\end{tabular}

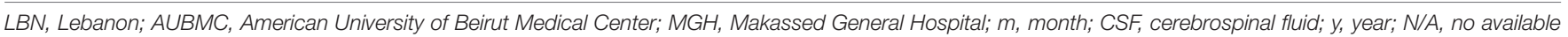
data.

Shimodaira-Hasegawa test implemented in FastTree2 was used to assess the support for significant clustering in the observed phylogeny.

\section{Genotypic Antibiotic Resistance Profile}

The isolates were analyzed for their penicillin-binding proteins (PBP) signature and GPSC number by uploading assembled genomes to Pathogenwatch ${ }^{2}$ where modifications of PBP to the native PBP1A, PBP2B, and PBP2X were also inspected. Antibiotic-resistance genes for all antibiotics were searched against a comprehensive antibiotic resistance database (CARD) using assembled genomes as input (Alcock et al., 2020).

\section{Virulence Gene Profile}

WGS was used to detect virulence factors such as pneumococcal surface protein A ( $p p A)$, pneumococcal surface protein C ( $p s p C / c b p A)$, pneumococcal adherence and virulence factor A (pavA and $p a v B)$, cell wall hydrolytic enzymes including lyt $A$, lyt $B$, lyt $C$, and pce (lytD and $c b p E$ ), neuraminidases $\mathrm{A}$, $\mathrm{B}$ and $\mathrm{C}$ (nan $A, \operatorname{nan} B$, and nan $C$, respectively), streptococcal enolase (eno), pneumococcal choline binding protein A ( $p c p A)$, pneumolysin ( $p l y)$, choline binding proteins including $c b p D$ and $\operatorname{cbp} G$ and pneumococcal serine-rich protein ( $p s r p$ ), the metal-ion-binding proteins ( $p s a A, c p p A, p i a A$, and piuA) as well as zinc metalloproteases (iga, $z m p B$, and $z m p C$ ), high temperature requirement $\mathrm{A}(h \operatorname{tr} A)$ and capsule related genes (cps). We used the VFanalyzer, an automatic and comprehensive platform for accurate bacterial virulence factors identification, downloaded from the Virulence Factors database (VFDB).

\section{Ethical Considerations}

Since data and samples from patients were collected prospectively through the IRB-approved LIPSP, and since the analysis of the bacteria was done in the absence of any patient identifiers, no additional ethical approval or informed consent from patients or guardians were required.

\footnotetext{
${ }^{2}$ https://pathogen.watch/
}

\section{Statistical Analysis}

Collected data were coded, introduced, and entered to the software Statistical Package for Social Sciences (SPSS) version 25 (SPSSTM Inc., Chicago, IL United States). At first, descriptive analyses were performed using numbers and percentages for qualitative variables and means with standard deviation (SD) for continuous variables. Second, the univariate analyses were conducted, Fisher's exact test was used for the comparison of percentages between two qualitative variables, in the event of an expected value $n<5$. A logistic regression model was carried out to identify the association between the time periods (before 2013, 2013-2018, and 2019) and the prevalence of serogroup/serotype 24. The strength of association was interpreted using the odds ratio (OR) with 95\% Confidence Interval (CI). A p-value $<0.05$ was considered statistically significant.

\section{RESULTS}

\section{Patient Characteristics}

We collected 8 isolates of serotype $24 \mathrm{~F}$ among a total of 587 invasive $S$. pneumoniae isolates, through the LIPSP from 2013 to 2019. Our findings showed a significant time effect where there was a significant increase of the prevalence of serotype $24 \mathrm{~F}$ observed over 3 different periods (period 1: before 2013, period 2: from 2013- to 2018, and period 3: 2019) with a $p$-value $<0.001$ and $\mathrm{OR}=8.837,95 \% \mathrm{CI}=(2.894-$ 26.985). The isolates were mainly recovered from children less than 6 years old (87.5\%). Males constituted $62.5 \%$ of the patients.

The source of the eight isolates were from the blood $(75 \%$, $n=6)$, followed by $\operatorname{CSF}(12.5 \%, n=1)$, and pleural fluid $(12.5 \%$, $n=1)$. Four isolates were recovered in 2019, while the others were isolated in 2013, 2017, and 2018 (Table 1). Pneumococcal vaccination status information was lacking although PCV-13 was introduced to the national immunization program in January 2016 to be given in three doses at 4, 6, and 12-15 months.

\section{Phenotypic Susceptibilities}

Using the latest CLSI breakpoints, the susceptibilities of the $S$. pneumoniae strains against 10 antibiotics are summarized 


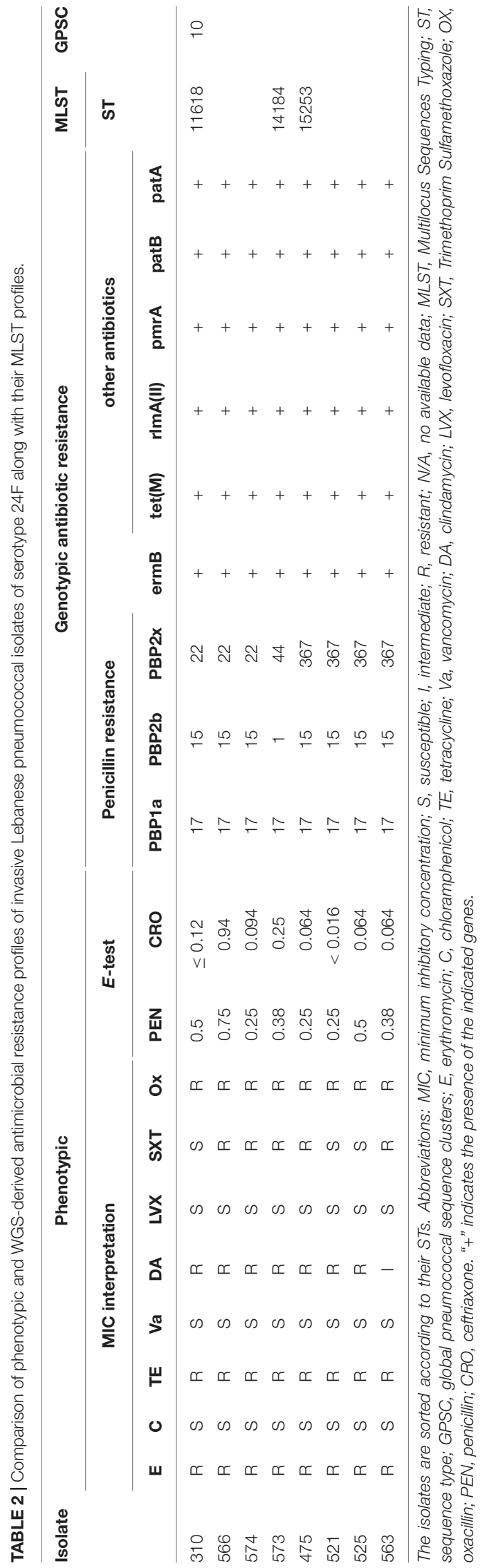

in Table 2. All the isolates were susceptible to penicillin except for 475 isolated from CSF and considered resistant. All of them were ceftriaxone, chloramphenicol, levofloxacin, and vancomycin sensitive. Resistance to tetracycline, erythromycin, clindamycin, and trimethoprim/sulfamethoxazole was $100 \%$ and 62.5 , respectively.

\section{Comparison of Phenotypic and Genotypic Antibiotic Resistance Profiles}

Resistance to $\beta$-lactams in S. pneumoniae occurs mainly through mutations in the genes coding for the PBPs essential for the bacterial cell wall synthesis. BLAST results of the PBPs protein types and the eight genomes showed that three isolates $(310,563$, and 574) had the 17 (PBP1a)-15 (PBP2b)-22 (PBP2x) signature explaining the penicillin susceptible phenotype predicted by the mode MIC (MM) model (Li et al., 2016), the other isolates showed new PBP protein type combinations 17 (PBP1a)-1 (PBP2b)-44 (PBP2x) (for the isolate 573) and 17 (PBP1a)15 (PBP2b)-367 (PBP2x) (for the isolates 475, 521, 525, and 563) (Table 2).

Moreover, for isolates showing erythromycin-clindamycin resistance, blast results in CARD showed the presence of $\operatorname{erm}(B)$ gene potentially responsible for the phenotypic resistance in these cases.

The eight isolates harboring the $\operatorname{erm}(\mathrm{B})$ gene, also harbored the tet(M) gene (Alcock et al., 2020). Other genes such as patA, pat $B, p m r A$, and $r \operatorname{lm} A(I I)$ encoding drug resistance mechanisms through efflux pumps and alteration of the antibiotic targets, respectively, were also detected. All the isolates harboring the $\operatorname{erm}(\mathrm{B})$ gene or tet(M) also harbored the mobile elements Tn6002 of the transposon Tn916 family (Table 2; Calatayud et al., 2010; Treangen et al., 2014; Akdogan Kittana et al., 2019). Moreover, tet $(\mathrm{M})$ and $\operatorname{erm}(\mathrm{B})$ were both carried on Integrative and conjugative element (ICE) ICESp12ST230 (Lo et al., 2020).

\section{Characterization of Pneumococcal Virulence Genes}

To gain insight into genetic features promoting virulence, we investigated the presence of major pneumococcal-protein virulence factors (Table 3 and Supplementary Table S1). Interestingly, when comparing our isolates to other S. pneumoniae isolates belonging to CC230, they share similar virulence genes profile. Among the virulence factors, cholinebinding proteins such as $c b p D, c b p G, l y t A, l y t B, l y t C$ and $p c e / c b p E, p s p A$, and $p s p C / c b p A$ were detected among all isolates in addition to pavA and $\operatorname{lm} b$ known as fibronectin and lamininbinding proteins, respectively. Hyaluronidase (hysA), nanA and streptococcal enolase (eno) as well as other genes known for their roles as iron and manganese uptake (piaA, piuA, and $p s a A$ and $c p p A$, respectively), IgA1 protease (iga), $z m p B$ and serine protease $(h t r A)$ were detected in all eight isolates, except for isolate 574 which lacks the piaA gene. For the capsule-related genes, $c p s A / B / C / D$ were detected among all isolates. However, none of the isolates harbored $z m p C$, pilus-1 ( $P I-1)$ related genes, or pilus-2 (PI-2) genes. Other virulence factors of adherence properties such as rlrA islet were not detected among these 
TABLE 3 | Virulence genes detected among Lebanese invasive pneumococcal isolates of serotype 24F.

\begin{tabular}{|c|c|c|c|c|c|c|c|c|c|}
\hline \multicolumn{2}{|c|}{ Genes } & \multicolumn{8}{|c|}{ Isolates } \\
\hline & & 310 & 475 & 521 & 525 & 563 & 566 & 573 & 574 \\
\hline \multirow[t]{9}{*}{ Adhesion } & $c b p D$ & + & + & + & + & + & + & + & + \\
\hline & $c b p G$ & + & + & + & + & + & + & + & + \\
\hline & lytA & + & + & + & + & + & + & + & + \\
\hline & lytB & + & + & + & + & + & + & + & + \\
\hline & lytC & + & + & + & + & + & + & + & + \\
\hline & $p c e / c b p E$ & + & + & + & + & + & + & + & + \\
\hline & $p s p A$ & + & + & + & + & + & + & + & + \\
\hline & $p s p C / c b p A$ & + & + & + & + & + & + & + & + \\
\hline & pavA & + & + & + & + & + & + & + & + \\
\hline \multirow[t]{3}{*}{ Enzyme } & hysA & + & + & + & + & + & + & + & + \\
\hline & $\operatorname{nan} A$ & + & + & + & + & + & + & + & + \\
\hline & eno & + & + & + & + & + & + & + & + \\
\hline Anti-phagocytosis & $c p s(A / B / B / D)$ & + & + & + & + & + & + & + & + \\
\hline \multirow[t]{2}{*}{ Iron uptake } & piaA & + & + & + & + & + & + & + & - \\
\hline & piuA & + & + & + & + & + & + & + & + \\
\hline Manganese uptake & psaA & + & + & + & + & + & + & + & + \\
\hline \multirow[t]{5}{*}{ Protease } & сррА & + & + & + & + & + & + & + & + \\
\hline & iga & + & + & + & + & + & + & + & + \\
\hline & htrA/degP & + & + & + & + & + & + & + & + \\
\hline & tig/ropA & + & + & + & + & + & + & + & + \\
\hline & $z m p B$ & + & + & + & + & + & + & + & + \\
\hline Toxins & ply & + & + & + & + & + & + & + & + \\
\hline
\end{tabular}

The present table shows the absence (-) or presence (+) of each considered genes from the VirulenceFactors database using VFanalyzer.

strains except those belonging to CC162 and CC72 mainly found in United Kingdom.

\section{Phylogenetic Analysis}

Three ST were identified (ST11618, ST14184, ST15253). Analysis of ST and assignment to clonal complexes (CCs) were performed using goeBURST (Figure 1A; Francisco et al., 2009). goeBURST full minimum spanning tree (MST) showing relationships among S. pneumoniae serotype 24 STs compared to serotype 24's available in the global PubMLST database revealed that the ST11618 and ST14184 originated from ST230. ST11618 differs in one single allele (aroE) from ST230 while ST14184 differs in two alleles ( $g d h$ and $d d l$ ). ST15253 originated from ST4253 and differs also in two alleles (aroE and spi) from ST230. Population snapshot of $412 \mathrm{~S}$. pneumoniae isolates of serogroup 24 using goeBURST full MST algorithm was added as Supplementary Figure S1.

Phylogenetic tree constructed from SNPs of representative 46 strains available in the global PubMLST database showed that among the 54 isolates, 11 CCs were identified (3 CCs PreVac, 6 CCs PostVac, 1 CC in both periods) (Figure 1B). The full data of these strains are presented in the Supplementary Table S2. The Lebanese isolates included in the study belonged to CC230 which emerged after PCV with three different STs. Isolates $(475,521,525$, and 563) having same ST15253 clustered and matched exactly with a Canadian isolate (PubMLST ID:51437). The other 3 isolates (566, 310, and
574) having same ST11618 clustered together with an isolate from Iceland (ST230); whereas, the isolate 573 having ST14184 did not cluster with any Lebanese isolate. Moreover, our isolates belonged to GPSC10, which is a common lineage of CC230 isolates.

\section{DISCUSSION}

The vaccine-related IPD incidence has dropped substantially by the introduction of PCVs. Nonetheless, non-PCV serotypes incidence is raising global concerns. So far, this is the first report in the Middle East and North Africa to describe the complete genome sequences of the emerging NVT 24F causing IPD in Lebanon. Serotype $24 \mathrm{~F}$ isolates represent $1.36 \%$ of all the surveillance isolates. These isolates were mainly recovered from children less than 6 years of age during the period 2013-2019.

Among NVTs, serotype 24F is considered one of the most prevalent causative agents of IPD in Europe and the Western Pacific Region (Balsells et al., 2017; Kawabata et al., 2020). 24F, collected as part of the French national survey program of pneumococcal infections, showed the highest invasive disease potential during the PCV13 period in children $<2$ years old (Varon et al., 2015). Furthermore, serotype 24F and other NVT such as $8,12 \mathrm{~F}$, and $33 \mathrm{~F}$ were considered at the upper end of the invasiveness spectrum among children immunized with PCV (Balsells et al., 2018). 

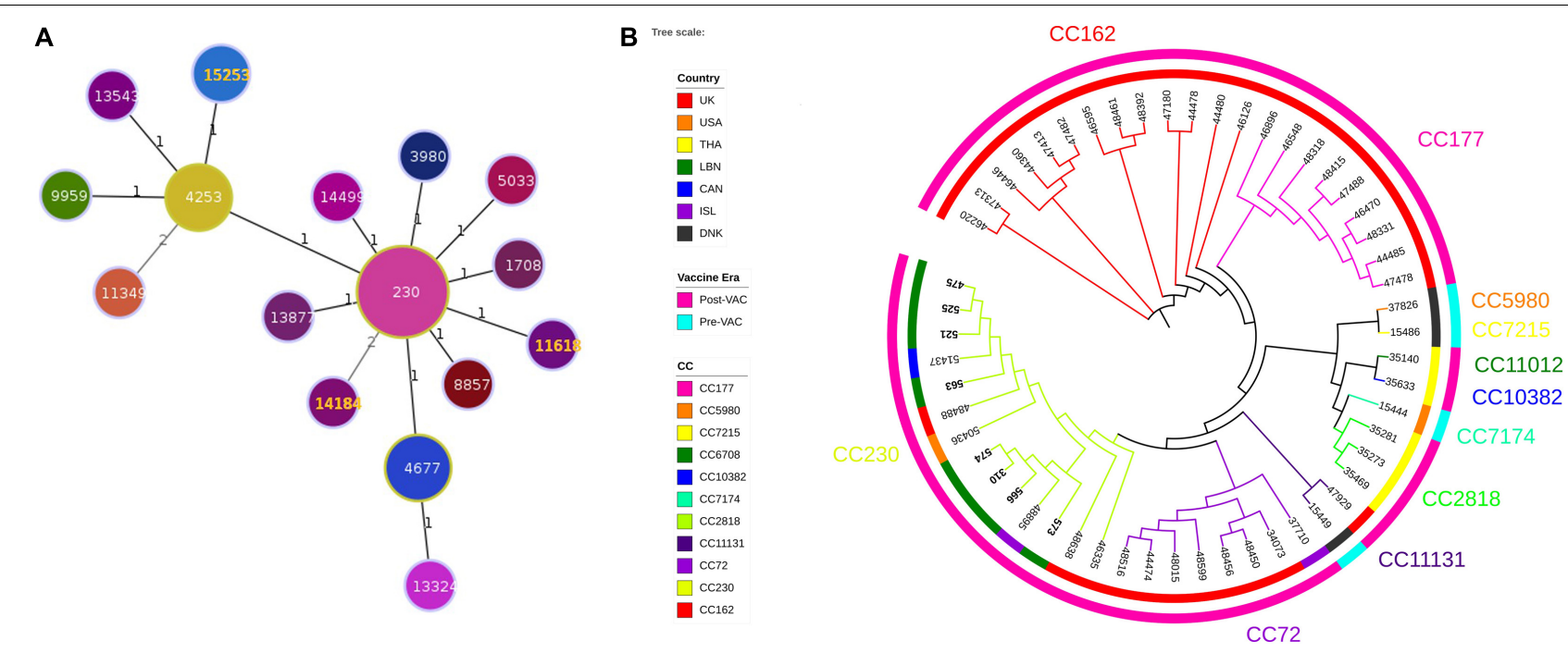

FIGURE 1 | (A) Minimum Spanning Tree (MST) analysis of serogroup 24 isolates. PHYLOViZ full MST algorithm presenting clonal complexes (CCs) using sequence types (STs) belonging to serotype 24 deposited in the PubMLST database as of 20 June 2020. STs are represented by circles proportional in size to the number of isolates. STs highlighted in orange corresponds to the Lebanese study isolates. Branch labels are the number of allelic variations between STs (number 1 represents single locus variant while number 2 represents double locus variants). STs links are grayscaled where darker links have less differences than the lighter gray links. (B) Whole genome SNP-derived phylogenetic tree. SNP-based phylogeny of 54 genomes, including 8 from the present study and 46 publically available closed genomes from the PubMLST database. Colored numbers presented on the outer most perimeter represents the MLST clonal complex of the isolates starting from its corresponding branches. Inner circle indicates the geographic origin of each strain: red: United Kingdom, orange: United States of America, yellow: Thailand, green: Lebanon, dark blue: Canada: purple: Iceland and black: Denmark. Outer circle is the study periods where cyan and fuchsia represent the pre- and post-vaccination periods, respectively. The leaf labels indicate the PubMLST isolates ID of the selected S. pneumoniae isolates.

Serotype $24 \mathrm{~F}$ isolates share the same ST (or CC) with other serotypes as shown in MLST database. Serotype $24 \mathrm{~F}$ belongs to the clonal complex CC230 among other serotypes including serotypes 14 and 19A which are also related to clone Denmark ${ }^{14}$-ST230. This lineage was reported to be largely distributed in southern part of Europe among children and adults in the PCV7 era (Aguiar et al., 2010). Likewise, 24F isolates belonging to CC156 with ST162, are correlated with $9 \mathrm{~V}$ serotype and related to clone Spain ${ }^{9 V}$-ST156 (Hanage et al., 2005; Sadowy et al., 2010; Kavalari et al., 2019). Both WGS and MLST typing confirmed that the Lebanese isolates were genetically homogeneous belonging to CC230 and clustered closely with isolates originating from Canada, United States, United Kingdom, and Iceland.

In $\beta$-lactam resistant $S$. pneumoniae isolates, the transpeptidase domains of PBPs were found to be altered thereby reducing their susceptibilities to cephalosporins and most penicillins ( $\mathrm{Li}$ et al., 2016). Our 24F isolates had the 17-15-x signature, except for the isolate (573) with 171-44 signature. Interestingly, isolates having the 17-15-22 signature share the same ST 11618, while those having the 17-15-367 signature share ST15253 and the one with the 17-1-44 signature has the ST14184. Lower-level $\beta$-lactam resistance have been associated with mutations within PBP2b and $\mathrm{PBP} 2 \mathrm{x}$, along with, additional changes within PBP1a essential for high-level resistance. The diversification of $p b p$ alleles is likely a consequence of transformation and homologous recombinational events among loci within S. pneumoniae and a number of closely related species resulting in a large pool of $p b p$ alleles with diverse $\beta$-lactam MICs (Dewe et al., 2019).

Macrolides are among the first line-agents for the treatment of penicillin-resistant pneumococcal infections. Globally, macrolide resistance is geographically variable ranging from $<10$ to $>90 \%$ of isolates (Schroeder and Stephens, 2016; El Moujaber et al., 2017). It is mainly driven via two mechanisms either by ribosomal dimethylation encoded by $\operatorname{erm}(\mathrm{B})$, efflux linked to the gene product of mef or due to mutations of the ribosomal target site of macrolides (Schroeder and Stephens, 2016). In this study, the $\operatorname{erm}(\mathrm{B})$ genes were present in all $24 \mathrm{~F}$ isolates, whereas none of the isolates had mef(A) or mef(E) suggesting that macrolide resistance is mainly mediated by the $\operatorname{erm}(\mathrm{B})$ gene. $\operatorname{erm}(\mathrm{B})$ was the most detected macrolide resistance determinant (36\%) among macrolide-resistant the strains followed by dual carriers of erm and mef genes (32\%), and by mef carriers (18\%) with $19 \mathrm{~F}$ being the most prevalent resistant serotype (Taha et al., 2012). Similarly, in Europe, the erm(B) gene is predominantly detected in France (87.5\%), Spain (77.3\%), Switzerland (80\%), and Poland $(100 \%)$, whereas the mef(A) gene is more common in Greece (100\%) and Germany (33.3\%) (Reinert et al., 2005). Association of macrolide and tetracycline resistance was reported with the acquisition of tet $(\mathrm{M})$ gene potentially through the conjugative transposon Tn6002 frequently encountered in Europe (Calatayud et al., 2010; Treangen et al., 2014; Akdogan Kittana et al., 2019). Additionally, tet(M) and erm(B) were both carried on ICESp12ST230 (Lo et al., 2020).

Moreover, other genes were detected among our isolates such as $p m r A$, a pneumococcal multidrug resistance gene, coding for 
an efflux pump as well as two $\mathrm{ABC}$ transporters pat $A$ and patB. Overexpression of these genes was reported to be linked directly with decreased fluoroquinolones susceptibility in clinical isolates of S. pneumoniae (El Garch et al., 2010).

The polysaccharide capsule has been considered a major virulence determinant factor in S. pneumoniae. Other proteins have also contributed to pathogenesis and known to be involved in the disease progression mainly through their interactions with the host defense mechanisms (Gamez et al., 2018). Our $24 \mathrm{~F}$ isolates carry a combination of virulence genes uniformly distributed among them; therefore, we did not find a correlation between virulence determinants distribution, antibiotic resistance genes and/or STs in our study. Finally, vaccines targeting non-serotype proteins are currently being studied as potential vaccine antigens. Among these, two were detected among our $24 \mathrm{~F}$ isolates such the PspC and the PspA. These approaches aim to display broader protective immunity and to provide coverage against all circulating pneumococcal strains (Garcia-Suarez Mdel et al., 2006; Chan et al., 2019).

\section{CONCLUSION}

In conclusion, we found a rise in serotype $24 \mathrm{~F}$ among patients with IPD in Lebanon. This is the first report in the Middle East and North Africa region that characterizes the full genomes of Streptococcus pneumoniae, serotype 24F. The studied isolates, collected from different regions in Lebanon, revealed similar antimicrobial resistance profiles and genetically homogenous patterns Continuous surveillance is emphasized in order to characterize emerging NVT at both national and regional levels and help improve knowledge to expand the vaccine polyvalency.

\section{DATA AVAILABILITY STATEMENT}

The genomes of $310,475,521,525,563,566,573$, and 574 have been deposited in GenBank under accession nos. CP046354, CP046355, CP046356, CP046357, CP046379, СP046358, CP046359, and CP046360, respectively.

\section{REFERENCES}

Aguiar, S. I., Pinto, F. R., Nunes, S., Serrano, I., Melo-Cristino, J., Sa-Leao, R., et al. (2010). Denmark14-230 clone as an increasing cause of pneumococcal infection in Portugal within a background of diverse serotype 19A lineages. J. Clin. Microbiol. 48(1), 101-108. doi: 10.1128/JCM.00665-09

Akdogan Kittana, F. N., Mustak, I. B., Hascelik, G., Saricam, S., Gurler, N., and Diker, K. S. (2019). Erythromycin-resistant Streptococcus pneumoniae: phenotypes, genotypes, transposons and pneumococcal vaccine coverage rates. J. Med. Microbiol. 68(6), 874-881. doi: 10.1099/jmm.0.000995

Alcock, B. P., Raphenya, A. R., Lau, T. T. Y., Tsang, K. K., Bouchard, M., Edalatmand, A., et al. (2020). CARD 2020: antibiotic resistome surveillance with the comprehensive antibiotic resistance database. Nucl. Acids Res. 48, D517-D525. doi: 10.1093/nar/gkz935.

Al-Sheikh, Y. A., Gowda K. L, Mohammed Ali, M. M., John, J., Khaled Homoud Mohammed, D., Chikkabidare Shashidhar, P. (2014). Distribution of serotypes and antibiotic susceptibility patterns among invasive pneumococcal diseases

\section{AUTHOR CONTRIBUTIONS}

GD and LR conceived and designed the study. IB, LR, JH, MF, and $M M$ conducted the experiments. AZ, CB, GA, GM, TJ, MN, $\mathrm{MH}, \mathrm{MO}$, and GK collected the samples. IB, LR, MF, and GD analyzed the data and wrote the manuscript. All authors revised and approved the final draft.

\section{FUNDING}

The authors declare that this study received funding from Pfizer (Grant No. WI227709). The funder was not involved in the study design, collection, analysis, interpretation of data, the writing of this article or the decision to submit it for publication. The study was also supported by the Charles University Research Fund PROGRES (project number Q39) and by the project No. CZ.02.1.01/0.0/0.0/16_019/0000787 Fighting Infectious Diseases provided by the Ministry of Education Youth and Sports of the Czech Republic.

\section{SUPPLEMENTARY MATERIAL}

The Supplementary Material for this article can be found online at: https://www.frontiersin.org/articles/10.3389/fmicb. 2021.637813/full\#supplementary-material

Supplementary Figure 1 | Population snapshot of 412 S. pneumoniae isolates of serogroup 24 using goeBURST full MST algorithm. STs that are linked by a line belong to the same cluster. Circle sizes are proportional to the number of isolates within the ST. STs links are grayscaled where darker links have less differences than the lighter gray links.

Supplementary Table 1 | Virulence factors distribution among Lebanese invasive pneumococcal isolates of serotype $24 \mathrm{~F}$ as well as 46 genomes available at the PubMLST database. VFanalyzer was used for the accurate bacterial virulence factors identification, downloaded from the VirulenceFactors database (VFDB: http://www.mgc.ac.cn/NFs/main.htm).

Supplementary Table 2 | A complete list of the 54 genomes including 8 from the present study and 46 publicly available closed genomes from the PubMLST database. This table includes the ID, country, year of isolation, diagnosis, source, serotype, STs, CCs, and the GPSCs of the corresponding S. pneumoniae isolates.

in Saudi Arabia. Ann. Lab. Med. 34, 210-215. doi: 10.3343/alm.2014.34.3. 210.

Ansaldi, F., Canepa, P., de Florentiis, D., Bandettini, R., Durando, P., and Icardi, G. (2011). Increasing incidence of Streptococcus pneumoniae serotype 19A and emergence of two vaccine escape recombinant ST695 strains in Liguria, Italy, 7 years after implementation of the 7 -valent conjugated vaccine. Clin. Vaccine Immunol. 18(2), 343-345. doi: 10.1128/CVI. 00383-10

Arbique, J. C., Poyart, C., Trieu-Cuot, P., Quesne, G., Carvalho Mda, G., Steigerwalt, A. G., et al. (2004). Accuracy of phenotypic and genotypic testing for identification of Streptococcus pneumoniae and description of Streptococcus pseudopneumoniae sp. nov. J. Clin. Microbiol. 42, 4686-4696. doi: 10.1128/JCM.42.10.4686-4696.2004

Bahy, R. H., Hamouda, H. M., Shahat, A. S., Yassin, A. S., and Amin, M. A. (2016). Emergence of Neoteric Serotypes Among Multidrug Resistant Strains of Streptococcus pneumoniae Prevalent in Egypt. Jundishapur. J. Microbiol. 9:e30708. doi: 10.5812/jjm.30708 
Balsells, E., Dagan, R., Yildirim, I., Gounder, P. P., Steens, A., Munoz-Almagro, C., et al. (2018). The relative invasive disease potential of Streptococcus pneumoniae among children after PCV introduction: A systematic review and meta-analysis. J. Infect. 77, 368-378. doi: 10.1016/j.jinf.2018.06.004

Balsells, E., Guillot, L., Nair, H., and Kyaw, M. H. (2017). Serotype distribution of Streptococcus pneumoniae causing invasive disease in children in the postPCV era: A systematic review and meta-analysis. PLoS One 12:e0177113. doi: 10.1371/journal.pone.0177113

Bentley, S. D., Aanensen, D. M., Mavroidi, A., Saunders, D., Rabbinowitsch, E., Collins, M., et al. (2006). Genetic analysis of the capsular biosynthetic locus from all 90 pneumococcal serotypes. PLoS Genet 2:e31. doi: 10.1371/journal. pgen.0020031

Brettin, T., Davis, J. J., Disz, T., Edwards, R. A., Gerdes, S., Olsen, G. J., et al. (2015). RASTtk: a modular and extensible implementation of the RAST algorithm for building custom annotation pipelines and annotating batches of genomes. Sci. Rep. 5:8365. doi: 10.1038/srep08365

Calatayud, L., Ardanuy, C., Tubau, F., Rolo, D., Grau, I., Pallares, R., et al. (2010). Serotype and genotype replacement among macrolide-resistant invasive Pneumococci in adults: mechanisms of resistance and association with different transposons. J. Clin. Microbiol. 48(4), 1310-1316. doi: 10.1128/JCM.018 68-09

Camacho, C., Coulouris, G., Avagyan, V., Ma, N., Papadopoulos, J., Bealer, K., et al. (2009). BLAST+: architecture and applications. BMC Bioinform. 10:421. doi: 10.1186/1471-2105-10-421

Carattoli, A., Zankari, E., Garcia-Fernandez, A., Voldby Larsen, M., Lund, O., Villa, L., et al. (2014). In silico detection and typing of plasmids using PlasmidFinder and plasmid multilocus sequence typing. Antimicrob. Agents Chemother. 58, 3895-3903. doi: 10.1128/AAC.02412-14

Chan, W. Y., Entwisle, C., Ercoli, G., Ramos-Sevillano, E., McIlgorm, A., Cecchini, P., et al. (2019). A Novel, Multiple-Antigen Pneumococcal Vaccine Protects against Lethal Streptococcus pneumoniae Challenge. Infect. Immun. 87, 846818. doi: 10.1128/IAI.00846-18

Clinical and Laboratory Standards Institute [CLSI] (2019). Performance standards for antimicrobial susceptibility testing; twenty-third informational supplement. M100-S29. Weinstein, MP: Clinical and Laboratory Standards Institute.

Croucher, N. J., Finkelstein, J. A., Pelton, S. I., Mitchell, P. K., Lee, G. M., Parkhill, J., et al. (2013). Population genomics of post-vaccine changes in pneumococcal epidemiology. Nat. Genet. 45, 656-663. doi: 10.1038/ng.2625

Daniels, C. C., Rogers, P. D., and Shelton, C.M. (2016). A Review of Pneumococcal Vaccines: Current Polysaccharide Vaccine Recommendations and Future Protein Antigens. J. Pediatr. Pharmacol. Ther. 21, 27-35. doi: 10.5863/15516776-21.1.27

Dewe, T. C. M., D’Aeth, J. C., and Croucher, N. J. (2019). Genomic epidemiology of penicillin-non-susceptible Streptococcus pneumoniae. Microb. Genom. 5:305. doi: 10.1099/mgen.0.000305

El Garch, F., Lismond, A., Piddock, L. J., Courvalin, P., Tulkens, P. M., and Van Bambeke, F. (2010). Fluoroquinolones induce the expression of pat $A$ and patB, which encode ABC efflux pumps in Streptococcus pneumoniae. J. Antimicrob. Chemother. 65, 2076-2082. doi: 10.1093/jac/dkq287

El Moujaber, G., Osman, M., Rafei, R., Dabboussi, F., and Hamze, M. (2017). Molecular mechanisms and epidemiology of resistance in Streptococcus pneumoniae in the Middle East region. J. Med. Microbiol. 66, 847-858. doi: 10.1099/jmm.0.000503

Francisco, A. P., Bugalho, M., Ramirez, M., and Carrico, J. A. (2009). Global optimal eBURST analysis of multilocus typing data using a graphic matroid approach. BMC Bioinform. 10:152. doi: 10.1186/1471-2105-10-152

Gamez, G., Castro, A., Gomez-Mejia, A., Gallego, M., Bedoya, A., Camargo, M., et al. (2018). The variome of pneumococcal virulence factors and regulators. BMC Genomics 19:10. doi: 10.1186/s12864-017-4376-0

Ganaie, F., Saad, J. S., McGee, L., van Tonder, A. J., Bentley, S. D., Lo, S. W., et al. (2020). A New Pneumococcal Capsule Type, 10D, is the 100th Serotype and Has a Large cps Fragment from an Oral Streptococcus. mBio 11, 937-20. doi: 10.1128/mBio.00937-20.

Garcia-Suarez Mdel, M., Vazquez, F., and Mendez, F.J. (2006). Streptococcus pneumoniae virulence factors and their clinical impact: An update. Enferm. Infecc. Microbiol. Clin. 24, 512-517. doi: 10.1157/13092469

Global Burden of Disease Study (2018). Estimates of the global, regional, and national morbidity, mortality, and aetiologies of lower respiratory infections in
195 countries, 1990-2016: a systematic analysis for the Global Burden of Disease Study 2016. Lancet Infect. Dis. 18, 1191-1210. doi: 10.1016/S1473-3099(18) 30310-4

Hanage, W. P., Kaijalainen, T. H., Syrjanen, R. K., Auranen, K., Leinonen, M., Makela, P. H., et al. (2005). Invasiveness of serotypes and clones of Streptococcus pneumoniae among children in Finland. Infect. Immun. 73, 431-435. doi: 10.1128/IAI.73.1.431-435.2005

Hanna-Wakim, R., Chehab, H., Mahfouz, I., Nassar, F., Baroud, M., Shehab, M., et al. (2012). Epidemiologic characteristics, serotypes, and antimicrobial susceptibilities of invasive Streptococcus pneumoniae isolates in a nationwide surveillance study in Lebanon. Vaccine 30, G11-G17. doi: 10.1016/j.vaccine. 2012.07.020

Hausdorff, W. P., Feikin, D. R., and Klugman, K. P. (2005). Epidemiological differences among pneumococcal serotypes. Lancet Infect. Dis. 5, 83-93. doi: 10.1016/S1473-3099(05)01280-6

Hicks, L. A., Harrison, L. H., Flannery, B., Hadler, J. L., Schaffner, W., Craig, A. S., et al. (2007). Incidence of pneumococcal disease due to non-pneumococcal conjugate vaccine (PCV7) serotypes in the United States during the era of widespread PCV7 vaccination, 1998-2004. J. Infect. Dis. 196, 1346-1354. doi: $10.1086 / 521626$

Isturiz, R. E., Hall-Murray, C., McLaughlin, J. M., Snow, V., Schmoele-Thoma, B., Webber, C., et al. (2018). Pneumococcal conjugate vaccine use for the prevention of pneumococcal disease in adults $<50$ years of age. Exp. Rev. Vacc. 17, 45-55. doi: 10.1080/14760584.2018.1411196

Janoir, C., Lepoutre, A., Gutmann, L., and Varon, E. (2016). Insight Into Resistance Phenotypes of Emergent Non 13-valent Pneumococcal Conjugate Vaccine Type Pneumococci Isolated From Invasive Disease After 13-valent Pneumococcal Conjugate Vaccine Implementation in France. Open Forum. Infect. Dis. 3:ofw020. doi: 10.1093/ofid/ofw020

Kapatai, G., Sheppard, C. L., Al-Shahib, A., Litt, D. J., Underwood, A. P., Harrison, T. G., et al. (2016). Whole genome sequencing of Streptococcus pneumoniae: development, evaluation and verification of targets for serogroup and serotype prediction using an automated pipeline. PeerJ 4:e2477. doi: 10.7717/peerj. 2477

Kavalari, I. D., Fuursted, K., Krogfelt, K. A., and Slotved, H. C. (2019). Molecular characterization and epidemiology of Streptococcus pneumoniae serotype $24 \mathrm{~F}$ in Denmark. Sci. Rep. 9:5481. doi: 10.1038/s41598-019-41983-8

Kawabata, T., Tenokuchi, Y., Yamakuchi, H., Sameshima, H., Katayama, H., Ota, T., et al. (2020). Concurrent Bacteremia Due to Non-vaccine Serotype 24F Pneumococcus in Twins: A Rapid Increase in Serotype 24F-invasive Pneumococcal Disease and its High Invasive Potential. Pediatr. Infect. Dis. J. 39, 85-87. doi: 10.1097/INF.0000000000002508

Kleinheinz, K. A., Joensen, K. G., and Larsen, M. V. (2014). Applying the ResFinder and VirulenceFinder web-services for easy identification of acquired antibiotic resistance and $\mathrm{E}$. coli virulence genes in bacteriophage and prophage nucleotide sequences. Bacteriophage 4:e27943. doi: 10.4161/bact.27943

Larsen, M. V., Cosentino, S., Rasmussen, S., Friis, C., Hasman, H., Marvig, R. L., et al. (2012). Multilocus sequence typing of total-genome-sequenced bacteria. J. Clin. Microbiol. 50, 1355-1361. doi: 10.1128/JCM.06094-11

Li, Y., Metcalf, B. J., Chochua, S., Li, Z., Gertz, R. E., Jr., Walker, H., et al. (2016). Penicillin-Binding Protein Transpeptidase Signatures for Tracking and Predicting beta-Lactam Resistance Levels in Streptococcus pneumoniae. mBio 7, 756-16. doi: 10.1128/mBio.00756-16.

Liu, B., Zheng, D., Jin, Q., Chen, L., and Yang, J. (2019). VFDB 2019: a comparative pathogenomic platform with an interactive web interface. Nucleic Acids Res. 47, D687-D692. doi: 10.1093/nar/gky1080.

Lo, S. W., Gladstone, R. A., van Tonder, A. J., Du Plessis, M., Cornick, J. E., Hawkins, P. A., et al. (2020). A mosaic tetracycline resistance gene tet(S/M) detected in an MDR pneumococcal CC230 lineage that underwent capsular switching in South Africa. J. Antimicrob. Chemother. 75, 512-520. doi: 10.1093/ jac/dkz477

Lo, S. W., Gladstone, R. A., van Tonder, A. J., Lees, J. A., du Plessis, M., Benisty, R., et al. (2019). Pneumococcal lineages associated with serotype replacement and antibiotic resistance in childhood invasive pneumococcal disease in the postPCV13 era: an international whole-genome sequencing study. Lancet Infect. Dis. 19, 759-769. doi: 10.1016/S1473-3099(19)30297-X

Matanock, A., Lee, G., Gierke, R., Kobayashi, M., Leidner, A., and Pilishvili, T. (2019). Use of 13-Valent Pneumococcal Conjugate Vaccine and 23-Valent 
Pneumococcal Polysaccharide Vaccine Among Adults Aged $>/=65$ Years: Updated Recommendations of the Advisory Committee on Immunization Practices. MMWR Morb. Mortal. Wkly. Rep. 68, 1069-1075. doi: 10.15585/ mmwr.mm6846a5

Mayanskiy, N., Savinova, T., Alyabieva, N., Ponomarenko, O., Brzhozovskaya, E., Lazareva, A., et al. (2017). Antimicrobial resistance, penicillinbinding protein sequences, and pilus islet carriage in relation to clonal evolution of Streptococcus pneumoniae serotype 19A in Russia, 20022013. Epidemiol. Infect. 145, 1708-1719. doi: 10.1017/S09502688170 00541

Moghnieh, R., Awad, L., Abdallah, D., Sleiman, R., Jisr, T., Tamim, H., et al. (2018). Epidemiology of pneumococcal infections in hospitalised adult patients in Lebanon with a highlight on non-invasive disease. J. Infect. Dev. Ctries 12:20S. doi: $10.3855 /$ jidc. 10106 .

Moghnieh, R., Tamim, H., Awad, L., Abdallah, D., Sleiman, R., Jisr, T., et al. (2020). Epidemiology of invasive and non-invasive pneumococcal infections in hospitalised adult patients in a Lebanese medical centre, 2006-2015. J. Infect. Public Health 13, 2092-2100. doi: 10.1016/j.jiph.2019.03.003

Mokaddas, E., and Albert, M. J. (2012). Impact of pneumococcal conjugate vaccines on burden of invasive pneumococcal disease and serotype distribution of Streptococcus pneumoniae isolates: an overview from Kuwait. Vaccine 30, G37-G40. doi: 10.1016/j.vaccine.2012.10.061

Munoz-Almagro, C., Ciruela, P., Esteva, C., Marco, F., Navarro, M., Bartolome, R., et al. (2011). Serotypes and clones causing invasive pneumococcal disease before the use of new conjugate vaccines in Catalonia, Spain. J. Infect. 63(2), 151-162. doi: 10.1016/j.jinf.2011.06.002

Namkoong, H., Ishii, M., Funatsu, Y., Kimizuka, Y., Yagi, K., Asami, T., et al. (2016). Theory and strategy for Pneumococcal vaccines in the elderly. Hum. Vaccin. Immunother. 12, 336-343. doi: 10.1080/21645515.2015.1075678

Ouldali, N., Levy, C., Varon, E., Bonacorsi, S., Bechet, S., Cohen, R., et al. (2018). Incidence of paediatric pneumococcal meningitis and emergence of new serotypes: a time-series analysis of a 16-year French national survey. Lancet Infect. Dis. 18, 983-991. doi: 10.1016/S1473-3099(18)30349-9

Pitsiou, G. G., and Kioumis, I. P. (2011). Pneumococcal vaccination in adults: does it really work? Respir. Med. 105, 1776-1783. doi: 10.1016/j.rmed.2011.07.008

Price, M. N., Dehal, P. S., and Arkin, A. P. (2010). FastTree 2-approximately maximum-likelihood trees for large alignments. PLoS One 5:e9490. doi: 10. 1371/journal.pone.0009490

Principi, N., Di Cara, G., Bizzarri, I., Isidori, C., Borgia, P., Mignini, C., et al. (2018). Prevention of Invasive Pneumococcal Disease: Problems Emerged After Some Years of the 13-Valent Pneumococcal Conjugate Vaccine Use. Curr. Infect. Dis. Rep. 20:1. doi: 10.1007/s11908-018-0607-z

Reinert, R. R., Ringelstein, A., van der Linden, M., Cil, M. Y., Al-Lahham, A., and Schmitz, F. J. (2005). Molecular epidemiology of macrolide-resistant Streptococcus pneumoniae isolates in Europe. J. Clin. Microbiol. 43, 1294-1300. doi: 10.1128/JCM.43.3.1294-1300.2005
Sadowy, E., Kuch, A., Gniadkowski, M., and Hryniewicz, W. (2010). Expansion and evolution of the Streptococcus pneumoniae Spain9V-ST156 clonal complex in Poland. Antimicrob. Agents Chemother. 54(5), 1720-1727. doi: 10.1128/AAC. 01340-09

Schroeder, M. R., and Stephens, D. S. (2016). Macrolide Resistance in Streptococcus pneumoniae. Front. Cell Infect. Microbiol. 6:98. doi: 10.3389/fcimb.2016. 00098

Taha, N., Araj, G. F., Wakim, R. H., Kanj, S. S., Kanafani, Z. A., Sabra, A., et al. (2012). Genotypes and serotype distribution of macrolide resistant invasive and non-invasive Streptococcus pneumoniae isolates from Lebanon. Ann. Clin. Microbiol. Antimicrob. 11:2. doi: 10.1186/1476-0711-11-2

Treangen, T. J., Ondov, B. D., Koren, S., and Phillippy, A. M. (2014). The Harvest suite for rapid core-genome alignment and visualization of thousands of intraspecific microbial genomes. Genome Biol. 15:524. doi: 10.1186/s13059014-0524-x

Varon, E., Cohen, R., Bechet, S., Doit, C., and Levy, C. (2015). Invasive disease potential of pneumococci before and after the 13-valent pneumococcal conjugate vaccine implementation in children. Vaccine 33, 6178-6185. doi: 10.1016/j.vaccine.2015.10.015

Vestrheim, D. F., Steinbakk, M., Aaberge, I. S., and Caugant, D. A. (2012). Postvaccination increase in serotype 19A pneumococcal disease in Norway is driven by expansion of penicillin-susceptible strains of the ST199 complex. Clin. Vaccine Immunol. 19, 443-445. doi: 10.1128/CVI.05563-11

Weinberger, D. M., Malley, R., and Lipsitch, M. (2011). Serotype replacement in disease after pneumococcal vaccination. Lancet 378, 1962-1973. doi: 10.1016/ S0140-6736(10)62225-8

World Health Organization (2007). Pneumococcal conjugate vaccine for childhood immunization - WHO position paper. Weekly Epidemiological Record = Relevé épidémiologique hebdomadaire, Geneva: World Health Organization, $82 \imath, 93-104$.

Zankari, E., Hasman, H., Cosentino, S., Vestergaard, M., Rasmussen, S., Lund, O., et al. (2012). Identification of acquired antimicrobial resistance genes. J. Antimicrob. Chemother. 67, 2640-2644. doi: 10.1093/jac/dks261

Conflict of Interest: The authors declare that the research was conducted in the absence of any commercial or financial relationships that could be construed as a potential conflict of interest.

Copyright (C) 2021 Reslan, Finianos, Bitar, Moumneh, Araj, Zaghlout, Boutros, Jisr, Nabulsi, Kara yaccoub, Hamze, Osman, Bou Raad, Hrabak, Matar and Dbaibo. This is an open-access article distributed under the terms of the Creative Commons Attribution License (CC BY). The use, distribution or reproduction in other forums is permitted, provided the original author(s) and the copyright owner(s) are credited and that the original publication in this journal is cited, in accordance with accepted academic practice. No use, distribution or reproduction is permitted which does not comply with these terms. 